\title{
Dual Ports L-Shaped Microstrip Antenna for WLAN Band Frequency
}

\author{
${ }^{1}$ Mohammed Qasim Abbas, ${ }^{1}$ Salam Ibrahim Kadhim, ${ }^{1}$ Omar Ibrahim Mustafa, ${ }^{1}$ Jinan F \\ Mahdi \\ \{Mohammadtoma44@gmail.com,Sal_yugo@yahoo.com,omdell_70@yahoo.com, \\ jinanf2008@yahoo.com \} \\ Middle Technical University ${ }^{1}$
}

\begin{abstract}
The future of mobile communications is still promising in which the reduced size of mobile handsets is a very attractive technology. MIMO antenna is a considerable design for such a system which required a small size, low mutual coupling, high gain and wide bandwidth. In this paper, the antenna model with dual-polarized ports of microstrip feeding lines with a radiator having an L-shape is proposed. Such a radiator geometry dimension is achieved at the WLAN operating frequency. The results confirm that the bandwidth frequency is $1.66(4.62-6.28) \mathrm{GHz}$ at $-10 \mathrm{~dB}$ along with an isolation approach $-15 \mathrm{~dB}$, while VSWR is not exceeded $2 \mathrm{~dB}$.
\end{abstract}

Keywords: Dual Ports, L-shaped, Microstrip Antenna, MIMO.

\section{Introduction}

The capacity of a wireless system in multipath environment can be enhanced by MIMO system [1]. Recently, the user equipment of Fourth Generation (4G) has been employed for MIMO system and promising to use such technology in future communications [2]. Accordingly, mitigation of multipath fading and increasing frequency spectrum without wasting resources can be achieved [3]. However, designers of MIMO antenna take into account antenna reducing size meanwhile eliminating the mutual coupling which stims from reducing the space between adjacent ports [4], [5]. On the other hand, a dual polarization is a technique that exploits to increase the bandwidth efficiency and consequently reducing the size of the prototype [6]. In order to achieve a compact model, easy fabrication and increasing the operating frequency bandwidth, incorporating more than one design method in MIMO antenna model such as modification of the radiator shape of patch microstrip feeding line [7]. Furthermore, to reduce the mutual coupling affected by the current surface, many techniques are used, such as parasitic geometry [8], metamaterial surface [9] and slitted pattern etched in the ground [10]. In [11], proposed a single port composite of right/left-handed patch antenna with a ground etched unit cell included a composite split resonator ring (CSRR) as a type of defected ground structure (DGS) in order to distribute radiation uniformly. Also, [12] presented a diversity MIMO system with dual ports, each one fed by a microstrip ended with L-shaped with hexagonal aperture etched in the ground resulted with UWB. This research aiming to more reducing size by proposing a dual-polarized MIMO antenna with patch microstrip L-shaped with CSRR to reduce the mutual and increase efficiency. 


\section{Design Single Element of MIMO Antenna}

The schematic diagram of the proposed antenna has shown in Figure 1. It consists of dual patch microstrip feeding lines ended by L-shaped as a radiator and connected to power with SMA connector. Such feeding lines are printed on the dielectric layer of FR-4 with a height of $1.6 \mathrm{~mm}, 4.4$ permittivity and $0.025 \tan \delta$. In order to achieve isolation between ports, a CRSS etched in the ground structure represented as a shunt LC. The geometry dimension details in Table1.

(a)

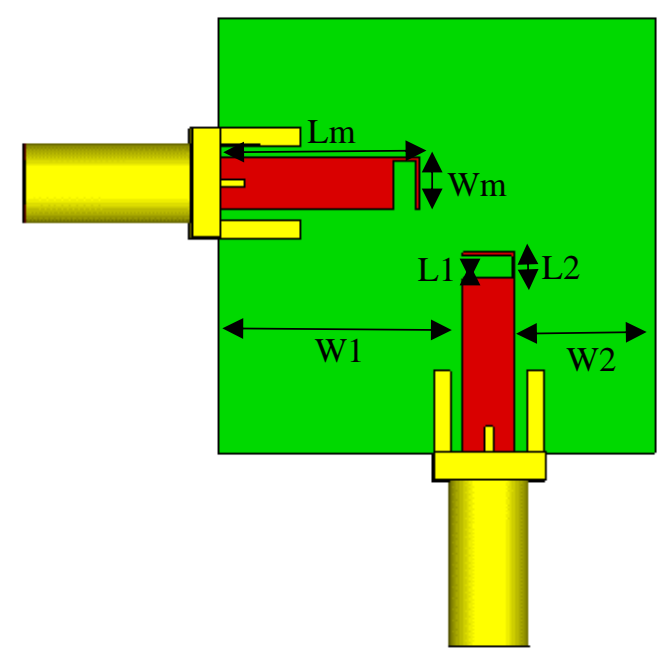

(a) (b)

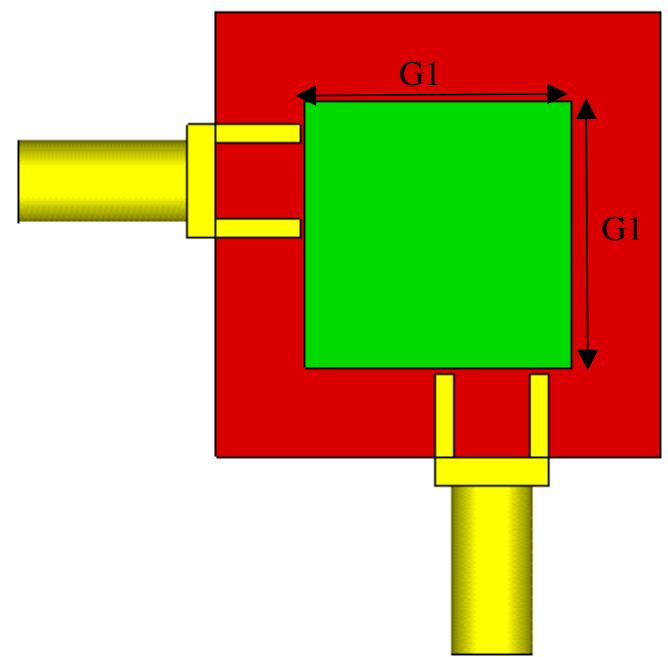

(b)

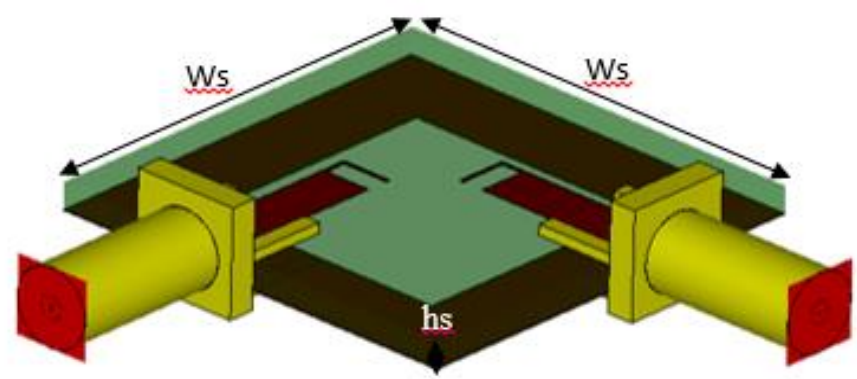

(c)

Fig.1. Single MIMO Antenna Geometry (a) Front View, (b) Back View and (c) Side View.

Table 1. MIMO Antenna Dimension.

\begin{tabular}{lcll}
\hline Parameter & Value $(\mathrm{mm})$ & Parameter & Value $(\mathrm{mm})$ \\
\hline $\mathrm{Lm}$ & 11.5 & $\mathrm{G} 1$ & 15 \\
$\mathrm{Wm}$ & 2.8 & $\mathrm{~W}_{\mathrm{s}}$ & 25 \\
$\mathrm{~L} 1$ & 1.3 & $\mathrm{~W} 1$ & 14.1
\end{tabular}




\section{Results and Discussions}

The proposed MIMO antenna model is simulated by CST-STUDIO 2019 covering the operating frequency $1.66(4.62-6.28) \mathrm{GHz}$ with an operating point of $5.04 \mathrm{GHz}$. Figure 2 shows the S-parameter results which illustrated $-25 \mathrm{~dB}$ for $S_{1,1}$ and $S_{2,2}$ and less than $-12 \mathrm{~dB}$ for mutual coupling between adjacent ports.

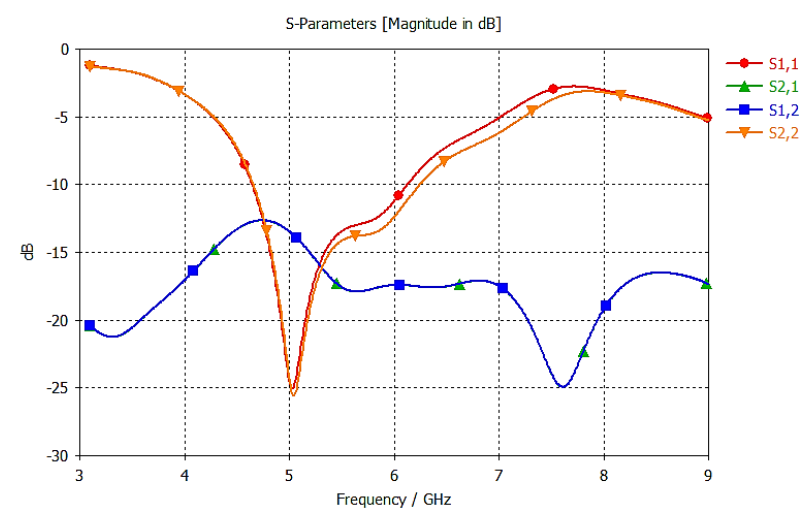

Fig. 2. S-Parameter of Single MIMO antenna element.

Also, VSWR which is the indication of antenna quality must be less than $2 \mathrm{~dB}$. Accordingly,

Figure 3 shows VSWR of the proposed antenna that is investigating such condition. 


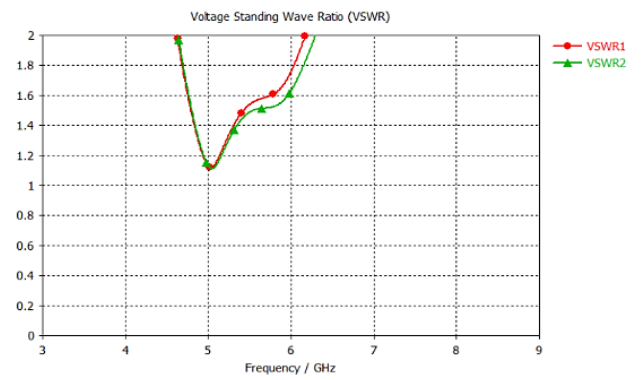

Fig. 3. VSWR of the Single MIMO antenna element.

On the other hand, the 2D radiation pattern of each port shows in Figure 4 which explicit that each one has radiated in bidirectional with the angular lobe of 69.5 and 70 degrees for port 1 and port 2, respectively. Meanwhile, when such ports operated simultaneously it obtained a 3D view of the radiation pattern in omnidirectional as well as covering the top and bottom of the printed circuit board (PCB) with H-max. $-29.67 \mathrm{~dB}$ and gain $4.4 \mathrm{dBi}$ as shown in Figure 5.

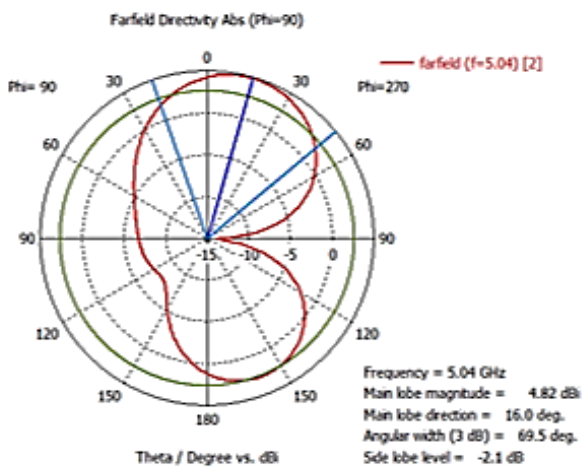

(a)

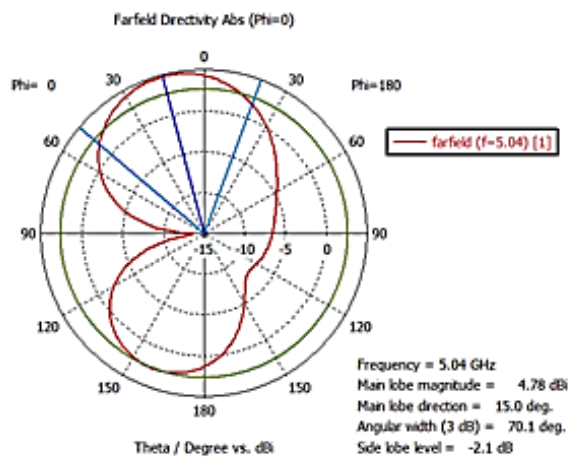

(b)

Fig. 4. 2D Radiation Pattern (a) Port1 and (b) Port2. 


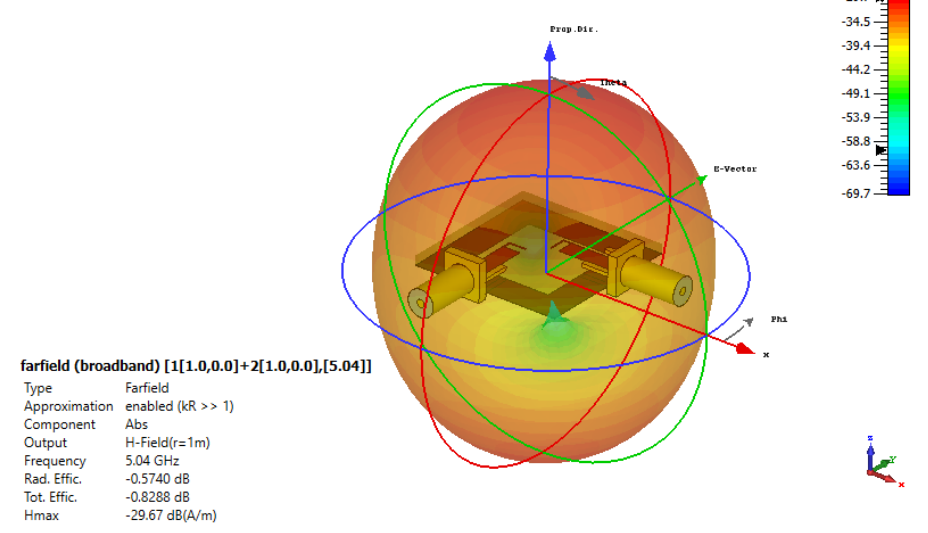

Fig. 5. 3D View of Radiation Pattern for both Ports.

In order to improve this proposed quality, Table 2 details a comparison between the current design and previous references. It is explicit that the current design having a small size with suitable return losses and isolation.

Table 2. Comparison Between Current Proposal and Previous References.

\begin{tabular}{cccccc}
\hline Ref. No. & $\begin{array}{c}\text { Element } \\
\text { Dimension }\end{array}$ & Return Losses & $\begin{array}{c}\text { Mutual } \\
\text { Coupling }\end{array}$ & $\begin{array}{c}\text { Operating Band } \\
(\mathrm{GHz}) \text { at }-10 \mathrm{~dB}\end{array}$ & Technique \\
\hline$[8]$ & $25 \times 25$ & $-20 \mathrm{~dB}$ & $-20 \mathrm{~dB}$ & $3.4-3.8$ & Polarization \\
{$[12]$} & $25 \times 25$ & $<-20 \mathrm{~dB}$ & $<-12 \mathrm{~dB}$ & $3.7-11$ & Spatial \\
{$[13]$} & $30 \times 30$ & $-50 \mathrm{~dB}$ & $<-20 \mathrm{~dB}$ & $8.13-10.19$ & Polarization \\
{$[14]$} & $100 \times 60$ & $-30 \mathrm{~dB}$ & $<-20 \mathrm{~dB}$ & $1.870-2.530$ & Spatial \\
{$[15]$} & $40 \times 20$ & $-55 \mathrm{~dB}$ & -13.5 & $1.7-3.66$ & Spatial \\
This & $25 \times 25$ & $-25 \mathrm{~dB}$ & $<-12 \mathrm{~dB}$ & $4.62-6.28$ & Polarization \\
Work & & & & & \\
\hline
\end{tabular}

\section{Conclusion}

A compact of dual-polarized antenna is proposed in this research in order to match the future wireless communication demands. The proposed model depends on the patch microstrip feeding line ended by L-shaped as a radiator. The results show that the resonance frequency of $5.04 \mathrm{GHz}$ with a bandwidth of $1.66 \mathrm{GHz}$ which is suitable for WLAN band frequency. Such a model can be used for small devices of wireless mobile applications. On the other hand, such a model can be extended to MIMO system for sub-band of $5 \mathrm{G}$ communication. 


\section{References}

[1] Q. U. A. Nadeem et al.: Design of 5G full dimension massive MIMO systems. IEEE Trans. Commun. pp. 726-740 (2018).

[2] A. Osseiran, et al.: Scenarios for 5G mobile and wireless communications: the vision of the METIS project. IEEE Commun. Mag. pp. 26-35 (2014).

[3] See, T. S. P., and Chen, Z. N.: An Ultra-wideband diversity antenna. IEEE Trans. Antennas Propag. pp. 1597-1605 (2009).

[4] J. L. Allen and B. L. Diamond: Mutual Coupling in Array Antennas. Lincoln Laboratory, M.I.T., Lexington, MA, Tech. Rep. 424 (ESD-TR-66-443) (1966).

[5] C. A. Balanis, Antenna Theory: Analysis and Design: Wiley, 1997.

[6] Shih-Ting Liu, Yao-Wen Hsu, and Yi-Cheng Lin.: A Dual Polarized Cavity-Backed Aperture Antenna for $5 \mathrm{G}$ mm W MIMO Applications. IEEE International Conference on Microwaves, Communications, Antennas and Electronic Systems (COMCAS)2015. pp. 1-5 (2015).

[7] Shereen Shandal, Yaqeen Mezaal, Mahmood Mosleh and Mohammed Kadim3: Miniaturized Wideband Microstrip Antenna for Recent Wireless Applications. Advanced Electronics. (2018).

[8] Naser Ojaroudi Parchin, Yasir Ismael Abdulraheem Al-Yasir, Ammar H. Ali, Issa Elfergani, James M. Noras , Jonathan Rodriguez and Raed A. Abd-Alhameed: Eight-ElementDualPolarized MIMO Slot Antenna System for 5G Smartphone Applications", IEEE Access. pp. 15612 - 15622 (2019)

[9] S. Gupta, Z. Briqech, A. R. Sebak, and T. A. Denidni: Mutual-coupling reduction using metasurface corrugations for $28 \mathrm{GHz}$ MIMO applications. IEEE Antennas Wireless Propag. Lett. pp. 2763-2766 (2017).

[10] C.-Y. Chiu, C.-H. Cheng, R. D. Murch, and C. R. Rowell: Reduction of mutual coupling between closely-packed antenna elements. IEEE Trans. Antennas Propag. pp. 1732-1738 (2007).

[11] Lin GENG, Guang-Ming WANG, Chen-Xin ZHANG, Xiang-Jun GAO, Bin-Feng ZONG: Compact Circularly Polarized Patch Antenna Using a Composite Right/Left-Handed Transmission Line Unit-Cell. Radio Engineering. (2013).

[12] Shobhit Saxena, Binod K. Kanaujia, Santanu Dwari, Sachin Kumar and Rahul Tiwari: A Compact Dual Polarized MIMO Antenna with Distinct Diversity Performance for UWB Applications. IEEE Antennas and Wireless Propagation Letters (2017).

[13] Nada M. Khalil Al-Ani, Oras A. Shareef Al-Ani, Mahmood F. Mosleh, Read A. AbdAlhameed,: A Design of MIMO System Based on YShaped with QSCS for UWB Applications. International Journal of Information Technology and Computer Science (IJITCS). pp.17-23 (2020).

[14] R.Hussain,A.T.Alreshaid,S.K.Podilchak,andM.S.Sharawi: Compact 4G MIMO antenna integratedwitha5Garrayforcurrentandfuturemobile handsets. IET Microw., Antennas Propag., pp. 271-279 (2017)

[15] Mohammed Alibakhshikhenari, et al.: A New Study to Suppress Mutual-Coupling between Waveguide Slot Array Antennas based on Metasurface Bulkhead for MIMO Systems. Proceedings of the 2018 Asia-Pacific Microwave Conference (APMC), pp.500-502 (2018). 